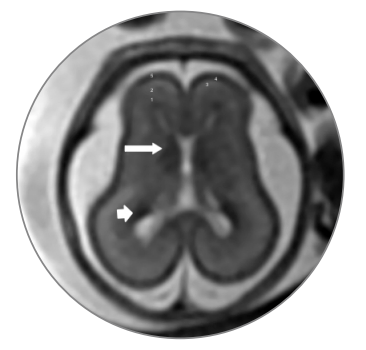

\section{Eminencia ganglionar: Anatomía y patología en resonancia magnética fetal}

\author{
Ganglionic Eminence: Anatomy and Pathology in Fetal MRI
}

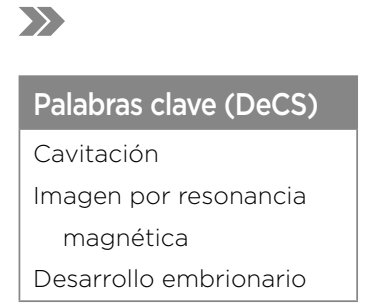

\section{Key words (MeSH)}

Cavitation

Magnetic resonance

imaging

Embryonic development
'Médico residente, Servicio de Diagnóstico por Imagen, Hospital Universitario Quirónsalud, Pozuelo de Alarcón. Madrid, España.

${ }^{2}$ Jefe asociado, Servicio de Diagnóstico por Imagen, Hospital Universitario Quirónsalud, Pozuelo de Alarcón. Madrid, España.

${ }^{3}$ Ginecóloga, Delta Ecografía. Madrid, España.

${ }^{4}$ Radióloga, Servicio de Diagnóstico por Imagen, Hospital Universitario Quirónsalud, Pozuelo de Alarcón. Madrid, España.

Servicio de Diagnóstico por Imagen. Hospital Universitario Quironsalud, Pozuelo de Alarcón. Madrid, España.

\title{
Resumen
}

Se presentan dos casos de resonancia magnética (RM) fetal en los que se detectan anomalías de las eminencias ganglionares (EG): un caso en una gestación única y otro en una gestación gemelar con solo uno de los fetos afectado. Las alteraciones en las eminencias ganglionares son entidades poco frecuentes, con muy pocos casos publicados, tanto por RM como por ecografía fetal, que suelen asociarse con alteraciones neurológicas graves. Se describen los hallazgos por RM de la patología de las EG en estos dos casos, no visibles en la ecografía previa.

\section{Summary}

We present two cases of fetal MRI where anomalies of the ganglionic eminences (GE) are detected, one case in a single pregnancy and another in a twin gestation with only one of the affected fetuses. Alterations in the ganglionic eminences are rare entities, with very few published cases, both by MRI and fetal ultrasound, which are usually associated with severe neurological alterations. The MR findings of the pathology of the GE in these two cases are described. These findings were not visible on the previous ultrasound.

\section{Introducción}

Las eminencias ganglionares (EG) son estructuras transitorias, proliferativas y embrionarias del telencéfalo ventral, que se localizan en la pared lateral de las astas frontales de los ventrículos laterales con leve extensión hacia las astas temporales (1). Las EG contienen los precursores neuronales de los ganglios basales y de las amígdalas, y proporcionan interneuronas que migran tangencialmente hacia la corteza, mediante el ácido $\gamma$-aminobutírico (GABA) como neurotransmisor principal (2).

La resonancia magnética (RM) fetal permite identificar estas estructuras, así como su patología - que se clasifica, según los hallazgos radiológicos, en EG cavitadas o EG prominentes-. A pesar de ser entidades muy poco frecuentes, es necesario familiarizarse con su diagnóstico prenatal.

\section{Descripción de los casos}

Paciente 1. Mujer de 35 años de edad con antecedente de cinco gestaciones y ningún embarazo a término. Los abortos fueron: uno causado por trisomía 21; uno, malformación cerebral compleja; dos, en el primer trimestre y uno, bioquímico. Se realizó ecografía en la semana 20 y 1 día que mostró hipoplasia cerebelosa y agenesia parcial del cuerpo calloso. Estudio genético con cariotipo convencional y molecular (array-CGH) normales.

Se realizó RM fetal a la edad gestacional de 20 semanas y 5 días, con hallazgo de prominentes EG en la matriz germinal, con cavitaciones bilaterales y simétricas y morfología en $\mathrm{C}$, sin evidencia de sangrado. No se identificó capa de neuronas intermedias entre la matriz germinal y la cortical externa inmadura, pero sí una prominente matriz germinal. Se observó horizontalización y engrosamiento de los pedúnculos cerebelosos superiores con malformación tipo "molar". Estos hallazgos no fueron visibles en la ecografía. Otros hallazgos asociados fueron: agenesia parcial del cuerpo calloso (ausencia de rodilla, esplenio y rostrum), marcada hipoplasia de ambos hemisferios cerebelosos y en menor medida del vermis; aumento del espacio subaracnoideo y leve colpocefalia, prominente cavum septum pellucidum, pequeño quiste del velum interpositum y engrosamiento del pliegue nucal, visibles en la ecografía. El resto del estudio fue normal (figura 1). La paciente decidió la interrupción voluntaria del embarazo sin consentimiento para la realización de necropsia.

Paciente 2. Mujer de 32 años de edad, primigestante de 26 semanas con gestación bicorial y biamniótica. Se le practicó ecografía en la semana 25 y 1 día con hallazgo de agenesia parcial del cuerpo calloso con ausencia de esplenio en uno de los fetos. Se le realizó una RM fetal a la edad gestacional de 26 semanas y 1 día. Se observaron prominentes EG y heterotopias periventriculares bilaterales asociadas a la alteración en el desarrollo cortical, con lisencefalia (cisuras de Silvio escasamente desarrolladas, sin signos de opercularización) y agenesia parcial del cuerpo calloso con ausencia del esplenio. El único patrón alterado en la biometría fetal fue el diámetro frontooccipital $(p<3)$ (figura 2). El otro feto no mostró alteraciones. 

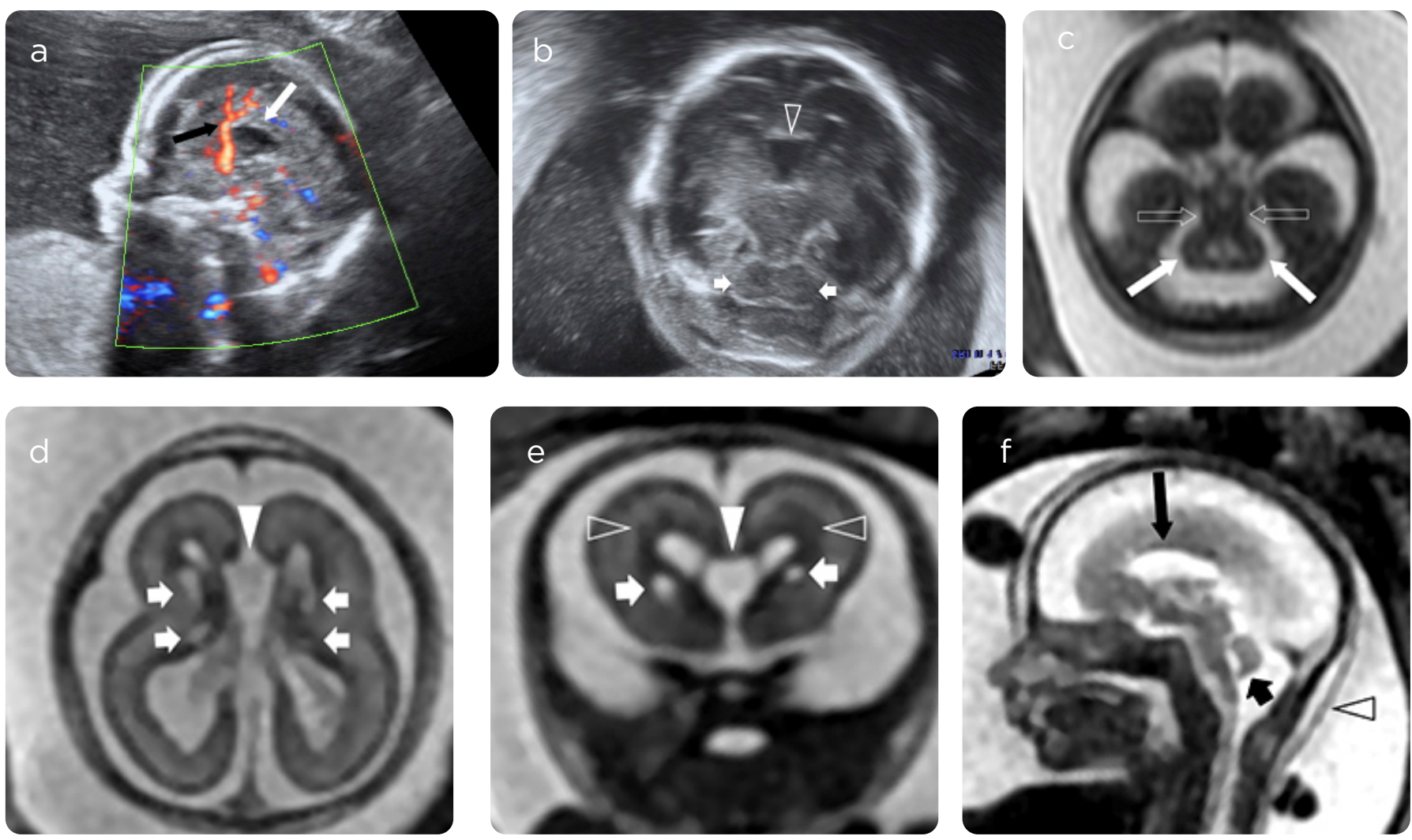

Figura 1. Cavitaciones bilaterales en eminencias ganglionares. Ecografía fetal. Edad gestacional 20 semanas y 1 día. a) Neurosonografía con plano medio sagital. Se identifica el tronco del cuerpo calloso (flecha blanca larga sólida) con ausencia de la rodilla y del esplenio. Las arterias pericallosas tienen una dirección craneal sin que describan el semicírculo típico alrededor del cuerpo calloso (flecha negra larga sólida). b) Neurosonografía con plano axial con hipoplasia de los hemisferios cerebelosos (flechas blancas sólidas). Diámetro transverso cerebeloso 15,8 mm. Area del hemisferio cerebeloso $40 \mathrm{~cm}^{2}$ y del hemisferio cerebeloso izquierdo $41 \mathrm{~cm}^{2}$. Prominente cavum septi pellucidi (punta de flecha blanca vacía). RM fetal. Edad gestacional 20 semanas y 5 días. c) Axial secuencia FIESTA. d) Axial e) Coronal f) Sagital secuencias SS FSE con información T2. Marcada hipoplasia de ambos hemisferios cerebelosos (flechas blancas largas sólidas) y en menor medida del vermis (flecha negra corta sólida). Horizontalización y engrosamiento de los pedúnculos cerebelosos superiores con imagen de malformación tipo molar (flechas blancas largas vacías). Prominente cavum septi pellucidi (punta de flecha blanca sólida). Microcefalia con prominentes eminencias ganglionares en la matriz germinal que presentan cavitaciones de forma bilateral y simétrica con morfología en C sin sangrado (flechas blancas cortas sólidas). No se identifica capa de neuromas intermedias entre la capa de neuronas de la zona ventricular que es muy prominente (puntas de flecha blanca vacías) y la cortical externa inmadura, futuras heterotopias en banda con microlisencefalia. Engrosamiento del pliegue nucal de $4,5 \mathrm{~mm}$ (punta de flecha negra vacía). Disgenesia de cuerpo calloso visualizándose el tronco del cuerpo de calloso (flecha larga negra sólida) con ausencia de su rodilla y esplenio
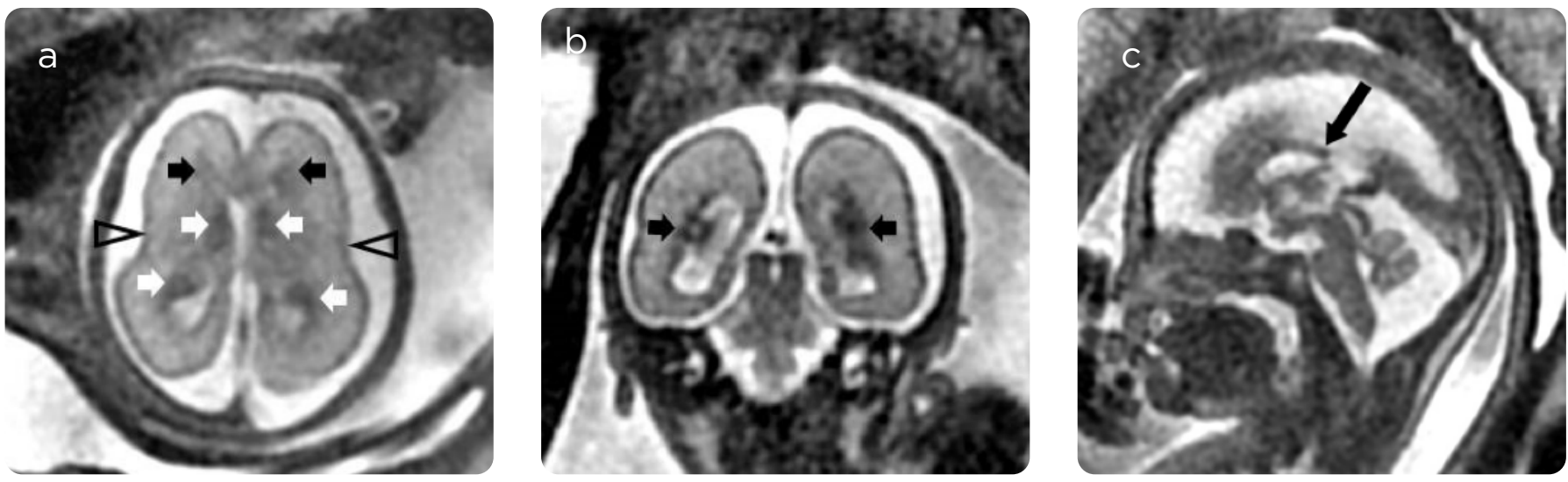

Figura 2. Aumento de tamaño de las eminencias ganglionares. Gestación bicorial y biamniótica de 26 semanas y 1 día. Feto patológico: a) axial b) coronal y c) Sagital secuencias SS y FSE con información T2. Prominentes eminencias ganglionares bilaterales (flechas blancas sólidas cortas) y prominente capa de neuronas periventriculares que no han migrado formando heterotopias subependimarias que marcan las paredes de los ventrículos laterales (flechas negras sólidas cortas). Disgenesia del cuerpo calloso cuya longitud es de $19,1 \mathrm{~mm}$ con ausencia del esplenio (flecha larga negra sólida). Escasa opercularización de las cisuras de Silvio (puntas de flechas negras vacías), la superficie cortical es lisa con ausencia del surco de Rolando. Tiene un diámetro frontooccipital cerebral de $63,3 \mathrm{~mm}$ que sugiere microlisencefalia. El cerebelo (no mostrado) es normal con un diámetro transverso cerebeloso de $29,8 \mathrm{~mm}$. 

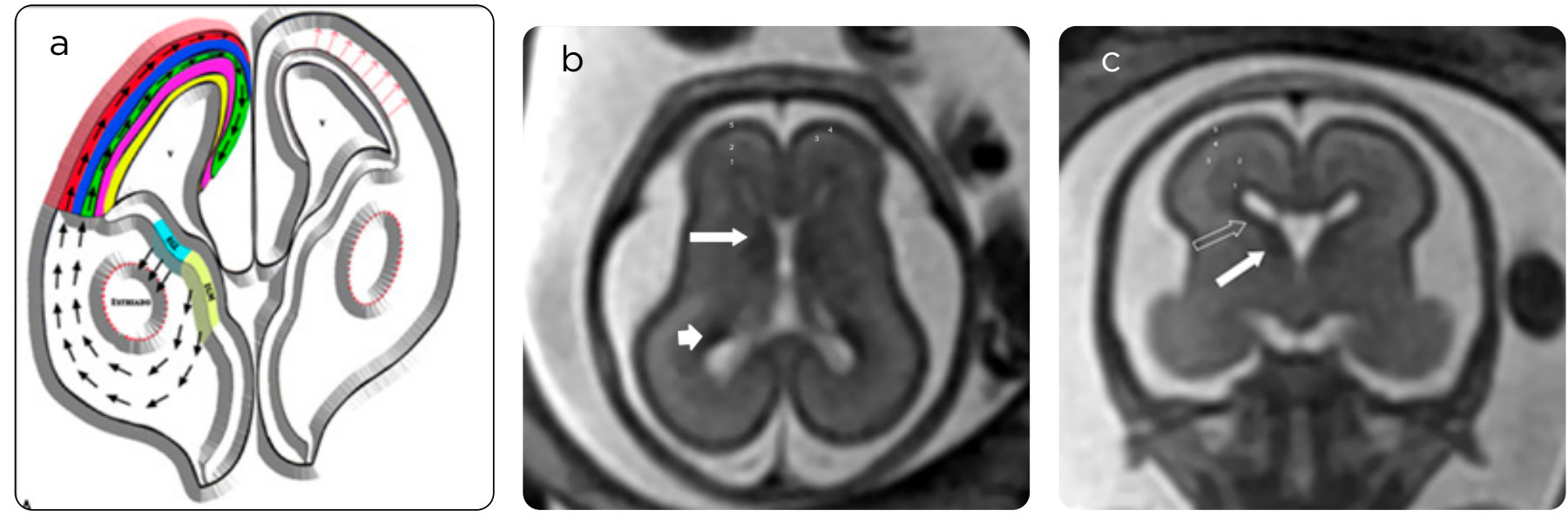

Figura 3. Anatomía normal y migración neuronal. Edad gestacional 22 semanas y 3 días. a) Esquema de migración neuronal: EGL: Eminencia Ganglionar Lateral. EGM: Eminencia Ganglionar Medial, V: Ventrículos. I Cortical externa inmadura. I Sustancia blanca subcortical. IZona de neuronas intermedias. Sustancia blanca periventricular. IZona neuronal ventricular. $\rightarrow$ Migración tangencial ...... Migración radial. b) Axial SS FSE T2. c) Coronal SS FSE T2. Se visualizan las cinco capas de esta fase de migración neuronal: (1) neuronas de la zona ventricular, (2) sustancia blanca periventricular, (3) capa de neuronas intermedias, (4) sustancia blanca subcortical y (5) cortical externa inmadura. Se identifican las eminencias ganglionares mediales (flechas blancas largas sólidas), eminencias ganglionares laterales (flechas blancas largas vacías) y eminencias ganglionares caudales (flechas blancas cortas sólidas).

\section{Discusión}

La corteza cerebral constituye la región más compleja del cerebro de los mamíferos y de ella emanan la mayor parte de las funciones que nos diferencian como seres humanos. Tradicionalmente, las neuronas corticales se han dividido en:

-1. Neuronas de proyección (excitadoras, glutaminérgicas)

- 2. Interneuronas (inhibidoras, gabaérgicas) (3).

Las neuronas de proyección se originan en la zona proliferativa del dorsopalio ventricular/subventricular y siguen una ruta migratoria radial (4). El $35 \%$ de las interneuronas que migran tangencialmente a la placa cortical proceden de la EG (derivadas del subpalio) (5).

La migración radial ocurre entre las semanas 12 y 16 y finaliza, aproximadamente, en la semana 24. Comienza en la zona germinal periventricular y termina en la superficie pial, con 6 capas sucesivas (6). La migración tangencial persiste más tiempo, termina después de la semana 24 (7).

La EG es una estructura embrionaria proliferativa y transitoria del telencéfalo ventral (subpalio) (3), que se localiza en la pared lateral de las astas frontales y en menor medida de las astas temporales de los ventrículos laterales (1). El subpalio se puede dividir en tres zonas proliferativas: eminencia ganglionar medial (EGM), eminencia ganglionar lateral (EGL) y eminencia ganglionar caudal (EGC), en función de las características anatómicas y genéticas (8). La EGL produce las interneuronas del bulbo olfatorio y las neuronas de proyección del núcleo estriado, la EGM y la EGC producen principalmente interneuronas corticales (9). Mientras que las células de la EGM migran lateralmente y se extienden por la corteza, la mayoría de las células de la EGC lo hacen inferiormente hacia el extremo más caudal del telencéfalo (8) (figura 3). El volumen de la EG aumenta con la edad gestacional, alcanza su máximo en las semanas 18-22 y disminuye significativamente en torno a la semana 30 (10). Persiste más tiempo que otras áreas proliferativas $\mathrm{y}$, normalmente, al nacimiento, ya ha desaparecido (11).
Recientemente, ha aumentado el interés sobre el origen y destino de las interneuronas, ya que se han relacionados ciertas patologías neurológicas y psiquiátricas - esquizofrenia, autismo, trastornos bipolares y encefalopatías epilépticas graves, como la asociada con el gen $\mathrm{ARX}$ - con la disminución de interneuronas gabaérgicas.

Riggini y colaboradores estudiaron mediante RM ocho casos de anomalía de las EG (excluyendo hemorragia), de los cuales tres presentaban EG prominentes y 5 cavitaciones en dicha localización $(10,11)$. Las cavitaciones fueron simétricas y bilaterales con una imagen similar a una "C abierta invertida" (11). En todos sus casos observaron lisencefalia con agenesia parcial o hipoplasia severa del cuerpo calloso, similar a los casos descritos en este artículo. Otros hallazgos fueron: hipoplasia y rotación del vermis, hipoplasia de hemisferios cerebelosos, ventriculomegalia, aumento del espacio subaracnoideo y malformación tipo "molar", presentes en nuestro primer caso. Al igual que en este estudio, las alteraciones en las EG no fueron visibles por ecografía.

El aumento del tamaño de EG puede estar asociado o no con cavitaciones $\mathrm{y}$, a la inversa, las cavitaciones pueden estar acompañadas o no con el aumento de tamaño de EG. Las hemorragias en las EG son lesiones irregulares y de alta señal en secuencias ponderadas en T1, lo cual es clave para distinguirlas de las cavitaciones (10). Se cree que es un proceso isquémico el responsable de la formación de estas cavitaciones y que la lisencefalia se produce por alteración de la migración neuronal (asumiendo que las cavitaciones actúan como barrera para impedir la correcta migración neuronal) (11). Esto respalda la idea de que las cavitaciones en la EG son parte de malformaciones complejas que involucran alteraciones en la migración neuronal.

En 2019, John Wiley publicó los dos primeros casos de cavitaciones EG visualizadas por ecografía prenatal (1). Hasta el momento no hay constancia de otras publicaciones que describan esta patología por RM.

\section{Conclusiones}

Las anomalías de las EG representan un hallazgo infrecuente que suele asociarse con alteraciones neurológicas severas. Es importante 
familiarizarse con los hallazgos radiológicos por RM fetal de esta patología, con el fin de facilitar su diagnóstico y ayudar al manejo y pronóstico fetal.

\section{Agradecimientos}

Especial mención y agradecimiento a Kenneth Estévez Hernández, por su colaboración en la realización del soporte gráfico.

\section{Referencias}

1. Prefumo F, Petrilli G, Palumbo G, Sartori E, Izzi C, Pinelli L. Prenatal ultrasound diagnosis of cavitation of ganglionic eminence. Ultrasound Obstetr Gynecol. 2019;54:558-60. doi: 10.1002/uog.20236

2. Wonders $C$, Anderson SA. Cortical interneurons and their origins. The Neuroscientist. 2005;11:199-205. doi:10.1177/1073858404270968

3. Marín O. Origen de las interneuronas de la corteza cerebral: conceptos básicos e implicaciones clínicas. Rev Neurol. 2002;35:743-51. doi: https://doi.org/10.33588/ rn.3508.2002460

4. Kolasinski J, Takahashi E, Stevens A, Benner T, Fischl B, Zöllei L, et al. Radial and tangential neuronal migration pathways in the human fetal brain: Anatomically distinct patterns of diffusion MRI coherence. NeuroImage. 2013;79:412-22. http://dx.doi. org/10.1016/j.neuroimage.2013.04.125

5. Zecevic N, Hu F, Jakovcevski I. Interneurons in the developing human neocortex. Develop Neurobiol. 2010;71:18-33. doi:10.1002/dneu.20812

6. Abdel Razek AA, Kandell AY, Elsorogy LG, Elmongy A, Basett AA. Disorders of cortical formation: MR imaging features. AJNR Am J Neuroradiol. 2009;30:4-11. doi:10.3174/ajnr.a1223

7. Arshad A, Vose L R, Vinukonda G, Hu F, Yoshikawa K, Csiszar A, et al. Extended production of cortical interneurons into the third trimester of human gestation. Cerebral Cortex. 2015;26:2242-56. doi:10.1093/cercor/bhv074

8. Yozu M, Tabata H, Nakajima K. The caudal migratory stream: A novel migratory stream of interneurons derived from the caudal ganglionic eminence in the developing mouse forebrain. J Neuroscience. 2005;25:7268-77. doi: 10.1523/jneurosci.2072-05.2005

9. Wei S, Du H, Li Z, Tao G, Xu Z, Song X, et al. Transcription factors Sp8 and Sp9 regulate the development of caudal ganglionic eminence-derived cortical interneurons. J Comparative Neurol. 2019;527:2860-74. doi: 10.1002/cne.24712

10. Righini A, Cesaretti C, Conte G, Parazzini C, Frassoni C, Bulfamante G, et al. Expanding the spectrum of human ganglionic eminence region anomalies on fetal magnetic resonance imaging. Neuroradiology. 2015;58:293-300. doi: 10.1007/s00234-015-1622-5

11. Righini A, Frassoni C, Inverardi F, Parazzini C, Mei D, Doneda C, et al. Bilateral cavitations of ganglionic eminence: A fetal MR imaging sign of halted brain development. AJNR Am J Neuroradiol. 2013;34:1841-5. http://dx.doi.org/10.3174/ajnr.A3508

\section{Correspondencia}

Daniel Martín Rodríguez

C/ Diego de Velázquez 1, Pozuelo de Alarcón, 28233

Madrid, España.

danielmr92@gmail.com

Recibido para evaluación: 19 de septiembre de 2020

Aceptado para publicación: 17 de diciembre de 2020 